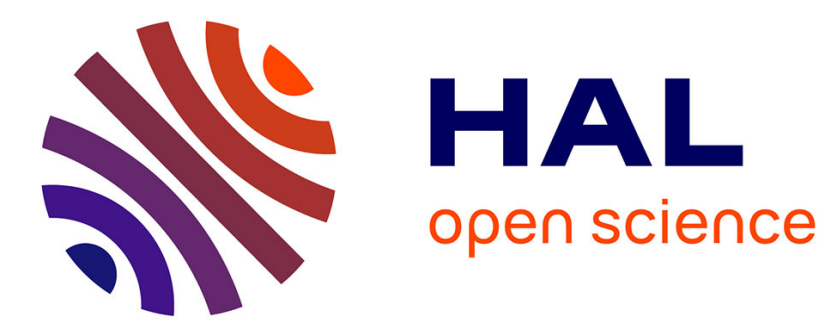

\title{
Challenges of Video Monitoring for Phenomenological Diagnostics in Present and Future Tokamaks
}

\author{
Vincent Martin, Victor Moncada, Jean-Marcel Travere
}

\section{To cite this version:}

Vincent Martin, Victor Moncada, Jean-Marcel Travere. Challenges of Video Monitoring for Phenomenological Diagnostics in Present and Future Tokamaks. 2011. hal-00609877

\section{HAL Id: hal-00609877 https://hal.science/hal-00609877}

Preprint submitted on 20 Jul 2011

HAL is a multi-disciplinary open access archive for the deposit and dissemination of scientific research documents, whether they are published or not. The documents may come from teaching and research institutions in France or abroad, or from public or private research centers.
L'archive ouverte pluridisciplinaire HAL, est destinée au dépôt et à la diffusion de documents scientifiques de niveau recherche, publiés ou non, émanant des établissements d'enseignement et de recherche français ou étrangers, des laboratoires publics ou privés. 


\title{
Challenges of Video Monitoring for Phenomenological Diagnostics in Present and Future Tokamaks
}

\author{
Vincent Martin, Victor Moncada, and Jean-Marcel Travere \\ CEA, IRFM, F-13108 Saint Paul Lez Durance, France. \\ Email: vincent.martin@cea.fr
}

\begin{abstract}
With the development of heterogeneous camera networks working at different wavelengths and frame rates and covering a large surface of vacuum vessel, the visual observation of a large variety of plasma and thermal phenomena (e.g., hot spots, ELMs, MARFE, arcs, dusts, etc.) becomes possible. In the domain of machine protection, a phenomenological diagnostic is a key-element towards plasma/thermal event dangerousness assessment during real time operation. It is also of primary importance to automate the extraction and the storage of phenomena information for further off-line event retrieval and analysis, thus leading to a better use of massive image data bases for plasma physics studies. To this end, efforts have been devoted to the development of image processing algorithms dedicated to the recognition of specific events. But a need arises now for the integration of techniques developed so far in both hardware and software directions. We present in this paper our latests results in the field of real time phenomena recognition and management through our image understanding software platform. This platform has been validated on Tore Supra during operation and is under evaluation for the foreseen imaging diagnostic of ITER.
\end{abstract}

\section{INTRODUCTION}

The interest in direct imaging diagnostics in nuclear fusion devices has gained a lot of attention during the last decades. Initially dedicated to specific physics analysis such as thermal studies from targeted in-vessel components monitoring (e.g. divertor tiles), imaging diagnostics are now considered as fully required for both plasma control systems and safety plasma operations. As a consequence, present Tokamaks are equipped and/or upgraded with networks of infrared and visible video cameras. However, as monitoring possibilities grow, a new bottleneck occurs at the image processing level. Therefore, new methods must be developed to tackle both real-time raw data processing issues and off-line data management issues. From this initial report, hardware and software related R\&D tasks have been identified as one of the most important risks regarding the feasibility of the diagnostic [1]. Different levels of performance have then been defined to deal with measurement requirements. In this paper, we will focus on the first level - qualitative imaging - which aims at delivering information for operational and critical functions of the diagnostic such as detection and identification of abnormal events (e.g., hot spots, impact of fast particles, edge plasma instabilities, moving objects, etc.) without requiring absolute measurements such as true surface temperature [2]. To this end, automatic image understanding is a real challenge mixing plasma physics knowledge modeling, computer vision techniques and realtime data processing.

The first technical specifications concerning sensor performance planned for the ITER VIS/IR imaging system are very challenging, especially for the infrared (see [2] for details) where one mega pixel sensors capturing images at $500 \mathrm{~Hz}$ are expected. With 36 video cameras, the amount of raw video data generated at each plasma discharge will be in the order of several hundreds of gigabytes. This corresponds to a data flow of $600 \mathrm{Mbit} / \mathrm{s}$ per camera (with pixels of 12 bits depth).

In this paper, we fist review in section II the different techniques developed so far for phenomenon recognition then discuss the needs in both hardware and software developments for the imaging diagnostics of next devices like ITER. In section III, we present the architecture of the proposed system and detail our current achievements on Tore Supra Tokamak in section IV.

\section{Towards Phenomenological Diagnostics}

\section{A. Related Work}

The literature related to phenomenological studies in nuclear fusion devices by means of direct imaging diagnostics reveals two categories: phenomena related to (1) edge plasma events like MARFEs, ELMs, arcs, turbulence (e.g. blobs, filaments), particle transport (dusts), and (2) Plasma Facing Components (PFCs) thermal events caused by power deposit like transient hot spots, fatigue effects (e.g. aging) and eroded material redeposition. The former are mostly observed with visible video cameras with frame rate ranging from $50 \mathrm{~Hz}$ to $250 \mathrm{kHz}$. The latter relies on both visible and infrared video monitoring with slower frame rates (from $25 \mathrm{~Hz}$ to $100 \mathrm{~Hz}$ ). We have reported in Figure 1 a selection of existing imaging systems used for phenomenological studies according to their temporal sampling and to their spectral sensitivity. This figure suggests that most of the events may be detectable using visible and MWIR sensors, with a sampling rate about few hundreds of frames per second.

The study of edge plasma transient events like MARFEs and ELMs as in FTU [3], JET [4]-[6] and NSTX [7] and turbulence blob extraction [8] is mainly carried out with visible imaging diagnostics. Some results have also been obtained with infrared sensors as in W7-AS [9], JET [10] and Tore 


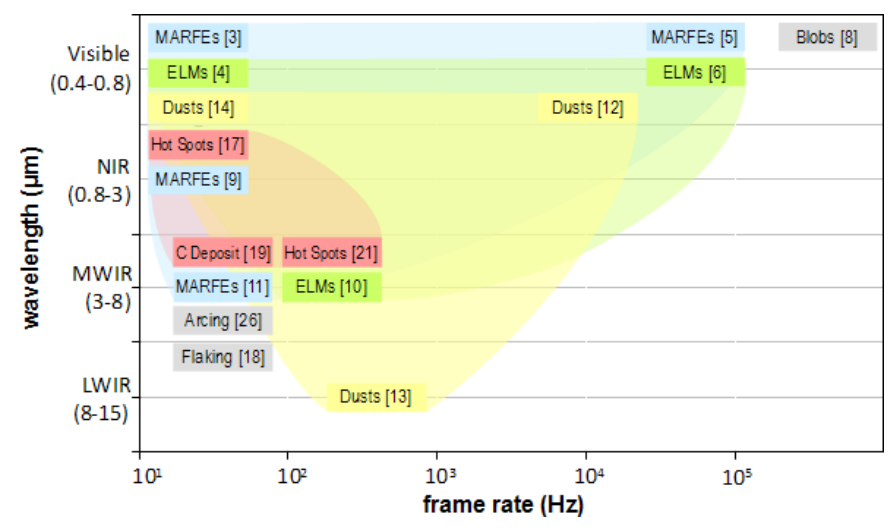

Fig. 1. Phenomenological diagnostic achievements depending on both temporal sampling and spectral sensitivity.

Supra [11]. In these works, rather than directly extracting physical quantities from images, the goal is to recognize visual patterns specific to an event (e.g. the apparent shape of a MARFE) by means of state-of-the-art image processing techniques. Since developed algorithms are quite robust to changes of plasma parameters, they can be applied to retrieve events in large data bases, paving the way to statistical analysis. Using fast imaging, dust particles can also be timeresolved and tracked during transport in the scape-off layer as demonstrated in NTSX [12] and in MAST [13] by means of stereoscopic systems. Then, Hong et al. [14] showed how long term dust event sampling from videos are used to figure out spatial distribution and density evolution of dust creation events. One can also point out the role of phenomenological information for better understanding of event interactions as done in [7] for MARFEs and ELMs.

Although qualitative imaging analysis is now widely used for plasma and thermal event recognition, it is worth mentioning that a complete event characterization often relies on the merging of information of different natures, e.g. image features and plasma parameters. The development of software tools dedicated to multi-sensor data analysis is therefore of primary importance. To this end, Martin et al. has proposed in [15] an integrated software platform gathering under the same graphical user interface common routines for multi-sensor data access, synchronization, manipulation, and visualization as well as specific functions for physics analysis purposes. This modular platform can be enriched with new algorithms that can be further easily shared and reused.

A main function of video monitoring in plasma fusion devices is dedicated to real time protection of PFCs. Systems developed so far are based on infrared imaging at Tore Supra [16] and visible imaging at AUG [17]. In the two cases, the goal is to trigger the power injection by real time detection of abnormal overheating of PFCs during plasma operation. To this end, a set of several regions of interest (RoIs) is defined for each monitored view and thresholds are set to maintain PFC surface temperature below material technological limits. Such systems are able to detect hot spots but do not aim at interpreting them. Off line image analysis are then often performed to understand the thermal behavior of the observed hot spots (see [18] and [19]) and to take adequate decisions as RoIs repositioning or changes in plasma heating schemes. A first step toward multi-event analysis has been proposed in [20] from Tore Supra infrared images. This cognitive vision system is able to recognize simultaneously several types of thermal events from a priori knowledge of event appearance (e.g. shape, size, etc.) and conditions of occurrence (e.g. plasma parameters). A real time implementation using hardwarebased acceleration has been proposed in [21]. An alternative approach has also been proposed in [22] for the real time detection of hot spots at JET.

\section{B. Challenges and Needs for Next Step Devices}

As a first report, the observation of edge plasma phenomenology by imaging diagnostics implies massive data production. To digest these vast amounts of data, efforts have been devoted to the development of image processing algorithms dedicated to the recognition of specific events. But a need arises now for the integration of techniques developed so far in both hardware and software directions. The next three sections describe the urgent needs that must be taken into account for efficient imaging data processing and management.

1) Data Storage and Retrieval: Image sensors commonly used for video monitoring in Tokamaks deliver uncompressed raw data. If the compression of imaging data is mandatory for long-term storage, the choice between lossless and loosely algorithms is not trivial and requires a performance assessment in terms of encoding/decoding speed versus compression rate versus signal-to-noise ratio. The efficient browsing of large image repositories from user-specific queries is also a key challenge. The storage of meta data as time stamp, event annotations and contextual information is then considered as an indispensable feature allowing quick image retrieval and event mining. To this end, content-based video indexing tools as proposed by Vega et al. in [23] must be developed and integrated into the data management system.

2) Integration of PFC Geometrical Models: Wide angle viewing systems (WAVS) are more and more used in Tokamaks to get a large coverage of the in-vessel components with a reduced number of sensors. One main issue when dealing with WAVS in Tokamaks is the high perspective of the observed scenes due to the toroidal geometry. The challenge is then to map each pixel from 2D planar Cartesian coordinates onto a 3D coordinate system based on the real positions of the in-vessel components. This preliminary task is particularly important in the case of infrared imaging to locate precisely detected hot spots on PFCs. The second interest in mapping pixels onto CAD-like models of PFCs is the possibility to index image content with meta-data describing the scene such as the registered names of observed components as well as material properties (e.g. emissivity factor) for measure calibration purposes. Then, image subparts showing only the studied component(s) can be loaded from the global image 
repository thus preserving network traffic from unnecessary overloads.

3) Real Time Processing: Real time video processing is a real challenge for machine protection functions because the performance level required for an accurate feedback control is typically in the range of hundred of milliseconds. The layered nature of an image processing work flow leads to design heterogeneous platforms with processing pipeline distributed over FPGA, CPUs and GPUs as seen in [21] and [22]. Intensive pixel-wise operations can be performed on FPGA providing the best robustness and performance levels while more complex treatments can be done on general purpose processing units as CPUs, more flexible in terms of developments. In all the cases, efficient data pipelining and high memory transfer rates are the key elements to reach highest performance levels. The architecture of such systems must then be thought to optimize the use of available data bandwidth of each processing unit.

\section{Proposed Approach OverviEW}

The synthesis of aforementioned experiences and first analysis of needs for future devices has motivated us to build up a new framework for phenomenological diagnostics based on video monitoring. Our main objective is to propose an heterogeneous architecture integrating both hardware and software components. This architecture will rely on multiple independent subsystems for modular, safe and reliable plasma / PFC monitoring, as seen in Figure 2 and summarized in Table I.

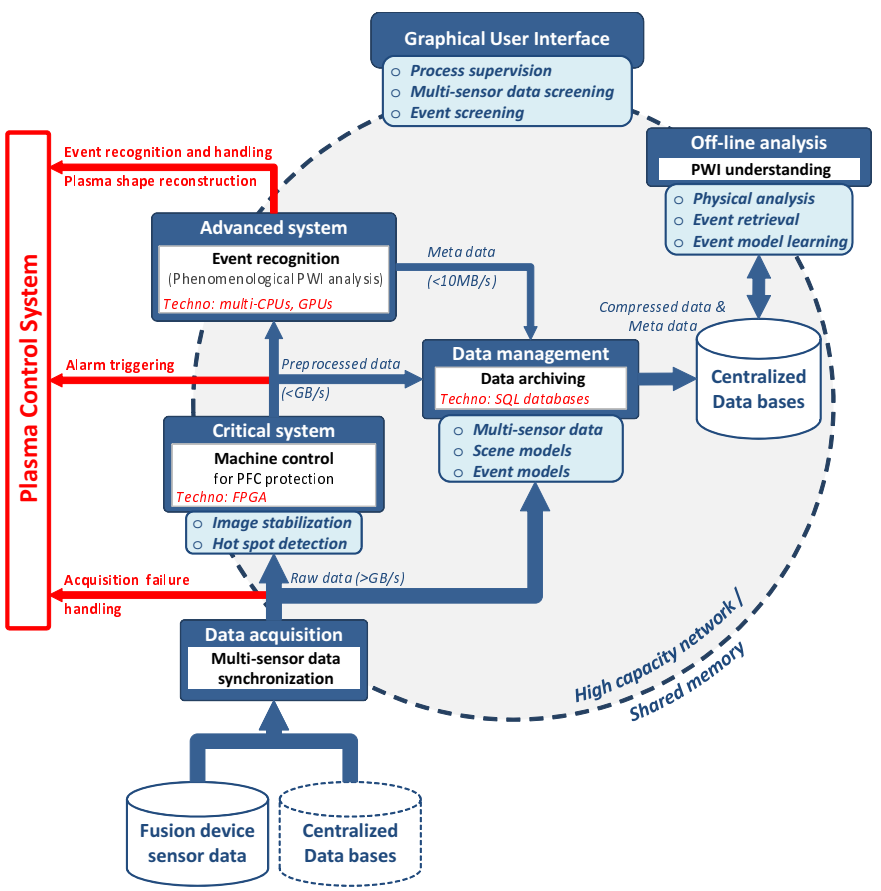

Fig. 2. Workflow of the proposed approach for phenomenological diagnostics based on video monitoring.

The critical system must achieve basic functions as the extraction of strike point position or alarm triggering from PFC overheating detection. This system, based on robust hardware components, must be designed to ensure a minimal safety level in case of failure(s) of more complex systems.

One prerequisite to RoI-based analysis is the scene motion compensation caused by camera or optical system shaking during strong mechanical stress of the device structures. This problem is often observed during plasma priming, transient events like ELMs and disruptions. As a consequence, RoI are misaligned thus leading to possible false alarms triggering or worst, to the miss-detection of true alarms. Automatic and real time image registration is then a mandatory feature that must be embedded in the critical system. Image processing needs rely mainly on pixel-wise operations as signal peak extraction. Such processing can be easily parallelized using hardware specific implementations as demonstrated in [21] to fulfill real time constraints of typically few milliseconds.

The proposed advanced system comes in addition to the critical system. Its main function is to handle alarms raised by the critical system based on event dangerousness evaluation for a more efficient plasma control. One typical action is to mitigate the possible false alarm raised by the detection of a hot spot. This can be done by analyzing its thermal behavior in order to discriminate between real PFC overheating and overheating due to e.g. poorly attached layers like heated carbon deposits. Since several events can occur simultaneously, CPU multi-threading based computing must be used to minimize the length of linear task sequences.

The data management module manipulates different types of data as raw images and meta-data as time window, bounding box coordinates, event annotations. Its main task is to prepare data for storage by compressing and time stamping of images as well as generating log files of detected alarms and event annotations. This module is also in charge of updating contextual information as scene models describing the positions of RoIs used for real time analysis.

In-between plasma discharge analysis happens within the off-line analysis module which is interfaced with the centralized data bases. This module provides tools to efficiently explore image repositories from user-defined semantic requests as the listing of plasma discharges where a specific event has been detected. Then, by studying the event conditions of occurrence, the performance of the algorithms used by the real time event recognition system can be benchmarked and optimized if needed.

The independent graphical user interface module is used to interface with each subsystem for parameter settings and for global system supervision. This graphical interface plays also a key role in the accurate live understanding of monitoring processes. One main feature is to help highlight and guide the user attention to abnormal video events detected from the camera network.

\section{Current Achievements}

A software platform named Plasma ImagiNg data Understanding Platform (PInUP) has been developed to fulfill a set of functions related to real time machine protection (e.g. 
TABLE I

SYSTEM REQUIREMENTS FOR PHENOMENOLOGICAL DIAGNOSTICS BASED ON VIDEO MONITORING.

\begin{tabular}{|c|c|c|c|}
\hline & \multicolumn{2}{|c|}{ Real Time Machine Protection and Control } & \multirow{2}{*}{ Edge Plasma Physics Studies } \\
\hline & Critical System & Advanced System & \\
\hline Functions & $\begin{array}{l}\text { PFC thermal load monitoring, } \\
\text { Strike point position tracking }\end{array}$ & $\begin{array}{l}\text { Plasma shape positioning, } \\
\text { Event handling (ELMs, MARFEs, arcs, hot spots), } \\
\text { Disruption precursors identification }\end{array}$ & $\begin{array}{l}\text { Particle transport analysis, } \\
\text { Edge turbulence characterization, } \\
\text { Runaway } \mathrm{e}^{-} \text {identification during } \\
\text { disruption }\end{array}$ \\
\hline Diagnostic & $\begin{array}{l}\text { Divertor viewing system (fast IR), } \\
\text { WAVS (VIS / IR) }\end{array}$ & $\begin{array}{l}\text { WAVS (fast VIS / IR), } \\
\text { Plasma scenario parameters }\end{array}$ & Stereoscopic WAVS (fast VIS) \\
\hline Input Data & $\begin{array}{l}\text { Raw images } \\
\text { Threshold maps }\end{array}$ & $\begin{array}{l}\text { Preprocessed images (e.g. filtered images) } \\
\text { Scene models } \\
\text { Event models (e.g. parameters for feature extraction } \\
\text { and pattern recognition) }\end{array}$ & $\begin{array}{l}\text { Compressed images, } \\
\text { Scene geometric calibration } \\
\text { matrices }\end{array}$ \\
\hline $\begin{array}{l}\text { Diagnostic } \\
\text { Features }\end{array}$ & $\begin{array}{l}\text { Alarm triggering on hot spot detection, } \\
\text { Signal peak position }\end{array}$ & $\begin{array}{l}\text { Transient event recognition (ELMs, MARFEs, arcs), } \\
\text { Plasma visible boundary reconstruction, } \\
\text { Hot spot characterization (deposits, flaking, cooling } \\
\text { trouble, impact of fast particles) }\end{array}$ & $\begin{array}{l}\text { Particle } 3 \mathrm{D} \text { trajectory } \\
\text { reconstruction, } \\
\text { Edge structure (filaments, blobs) } \\
\text { reconstruction }\end{array}$ \\
\hline $\begin{array}{l}\text { Image } \\
\text { Processing } \\
\text { Needs }\end{array}$ & $\begin{array}{l}\text { Image stabilization, } \\
\text { Pixel mapping onto real 3D geometry, } \\
\text { RoI-based analysis } \\
\text { Basic filtering operations }\end{array}$ & $\begin{array}{l}\text { Pattern recognition and tracking, } \\
\text { Multi-sensor data and event merging } \\
\text { (IR+VIS+plasma parameters) }\end{array}$ & $\begin{array}{l}\text { Particle detection, correspondence } \\
\text { solving and tracking }\end{array}$ \\
\hline $\begin{array}{l}\text { Computing } \\
\text { Performance } \\
\text { Requirements }\end{array}$ & $\begin{array}{l}\text { Fully autonomous embedded system } \\
\text { (e.g. FPGA) }\end{array}$ & $\begin{array}{l}\text { Pixel level parallelism (e.g. GPU) for low-Level } \\
\text { feature extraction, } \\
\text { Task-level parallelism (e.g. multi-cores CPUs) for } \\
\text { high-level reasoning algorithms }\end{array}$ & Fast distributed data access \\
\hline
\end{tabular}

automatic abnormal event recognition) and physical studies (e.g. off-line multi-sensor data analysis). This platform is daily used at Tore Supra to monitor plasma and PFCs from the infrared (7 cameras) and visible ( 2 cameras) viewing systems.

\section{A. Multi-Sensor Based Event Recognition}

One key issue of phenomenological diagnostics is to estimate a confidence level of their semantic outputs. Since events can generally be detected by several diagnostics (e.g. by IR and visible cameras), combining video events with other sensor information helps in achieving a robust diagnosis. This ability to correlate multi-sensor event is indeed considered as an essential element for plasma control system reliability in case of unexpected failure of one diagnostic during plasma operation. Our system is designed to perform such multisensor event fusion as illustrated in Figure 3 where we present the result of electrical arc event recognition using both IR and visible imaging diagnostics during the same plasma discharge at Tore Supra (see [24] for details). Although both sensors data and observed scenes are completely different, the system achieves the recognition of the same arc event appearing in front of the same RF heating antenna.

\section{B. Event Visualization and Management}

Figure 4 shows grabbed images from the graphical user interface of PInUP during a plasma discharge at Tore Supra. These images correspond to the live display of the infrared

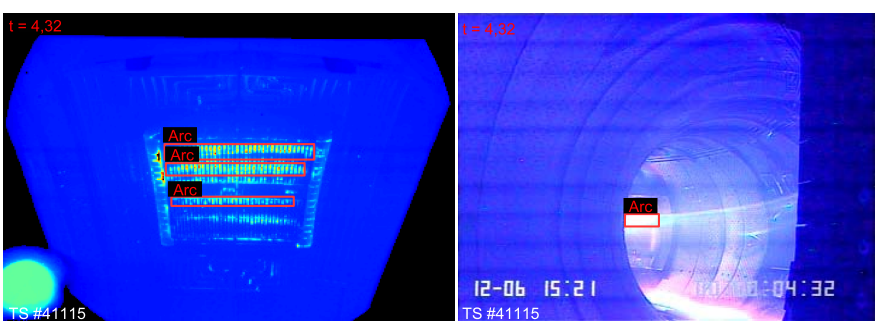

Fig. 3. Synchronized IR (left) and visible (right) camera views aiming the same PFC (heating antenna) from different lines of sight inside the Tore Supra tokamak. The arc event is successfully recognized by the system in the two cases.

view of the LHCD launcher with luminance temperature levels represented in gray levels. Each bounding box corresponds to the position of a detected hot spot with its associated event tag as reflection, arc, etc.

This synthesized display comes with a timeline of detected events represented in the bottom part of Figure 5. The goal of this timeline is to reconstruct the tracks of the detected events and to correlate them with plasma parameters as plasma current and injected power levels (top part of Figure 5) so as to ease the global understanding of the phenomenological activity detected from one or several monitored components. The meta-data of each detected event are then automatically stored in a centralized SQL data base. This lets the user the possibility to further retrieve and mine events for off-line 

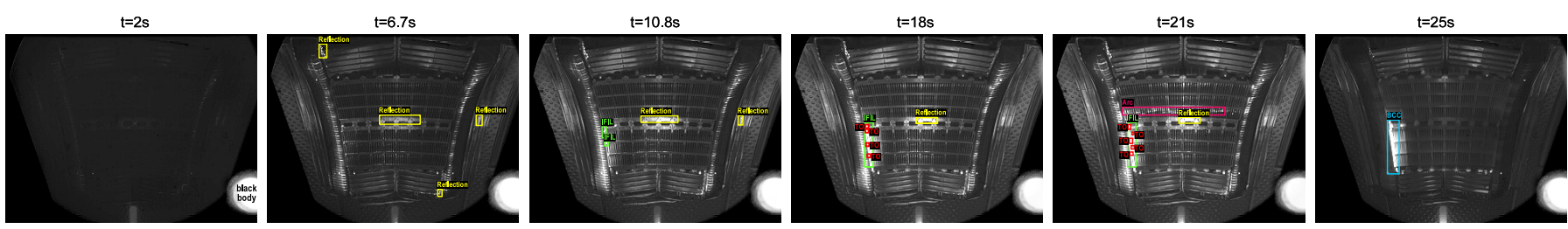

Fig. 4. Comprehensive display of recognized thermal events during a plasma discharge. Event patterns are superimposed on the infrared view of a LHCD launcher. TO stands for Threshold Overrun, IFIL stands for Impact of Fast Ion Losses and BCC stands for Bad Component Cooling. See Figure 5 for timeline correspondence.
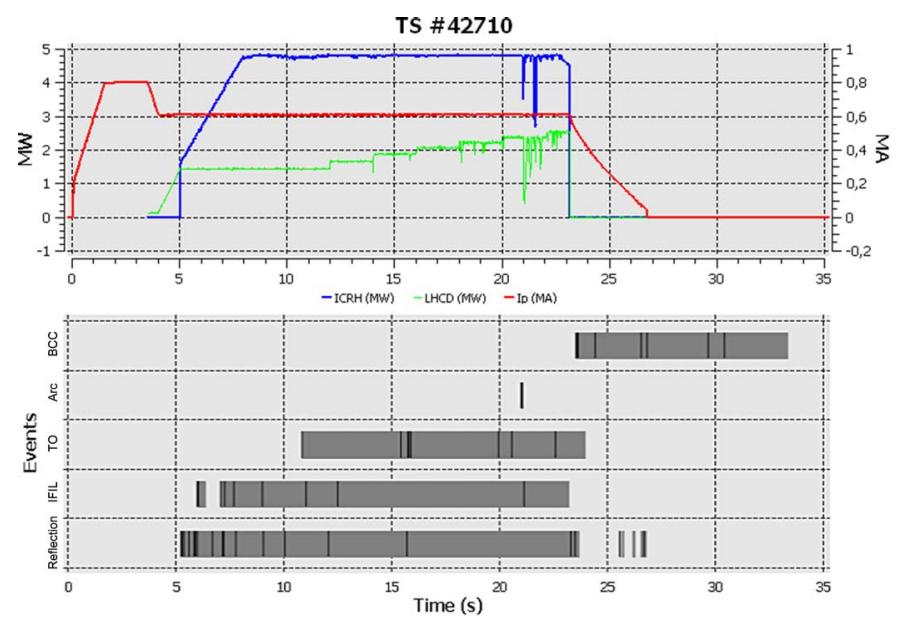

Fig. 5. Generated thermal event timeline during a plasma discharge (see Figure 4 for corresponding images). TO stands for Threshold Overrun, IFIL stands for Impact of Fast Ion Losses and BCC stands for Bad Component Cooling.

analysis purposes.

\section{Real time processing}

As explained in section III, some time-consuming tasks related to intensive pixel-level computations must be accelerated using specific hardware supporting high process parallelization. In our case, we have focus on the real time implementation of transient event detection algorithms using a FPGA (see [21] for details), hot spot detection using multi cores CPUs and image stabilization techniques using a GPU. These three algorithms have been chosen since they are identified as critical image processing needs for machine protection, as described in Table I). Concerning image stabilization, several techniques has been yet proposed to tackle this issue as in Alcator C-Mod [25] and MAST [26]. However, these techniques are either not adapted for real time computations or too brittle for noisy infrared images. To cope with these issues,we have developed a robust Fourierbased image registration algorithm able to detect and to quantify image geometric transformations (e.g. translations, homothety) with a sub-pixel precision. This algorithm has been implemented on a recent GPU (NVidia GTX 580) and its computational performance has been benchmarked on real infrared videos of Tore Supra and JET representing various operational conditions. Results reported in Figure 6(a) show that high frame rates ( $>1000 \mathrm{fps})$ can be achieved with a quarter pixel of precision using a $128 \times 128$ pixels sub-image. This first encouraging experience shows that there is still a considerable margin for dealing with higher performance as it is planned for the ITER VIS/IR diagnostic $(1000 \times 1000$ pixels at $500 \mathrm{~Hz}$ ). Actually, GPUs may be considered as an intermediate solution between FPGA and CPU in terms of computational performance and implementation complexity. Our second experience concerns the assessment of multi-cores CPUs platforms for high performance image processing. We have benchmarked a local adaptive thresholding algorithm used for the detection of hot spots based on local image contrast analysis. Depending on the number of available CPU core units, here between one and 24 cores from two AMD Opteron CPUs, the image is automatically split into a corresponding number of sub-images all processed in parallel. As seen in Figure 6(b), the computational speed increases quasi-linearly with the number of dedicated CPU cores. However, application on big images seems to be limited by the local bus bandpass, showing that memory management must be improved.

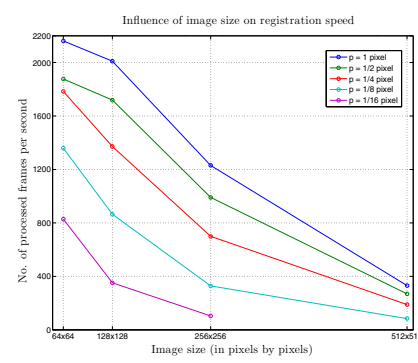

(a)

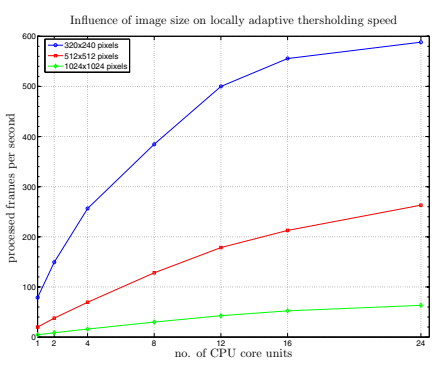

(b)
Fig. 6. (a) Influence of image size on image registration speed using a GPU. (b) Influence of the number of CPU core units on the image local thresholding speed.

\section{CONCLUSiON}

Next nuclear fusion devices like ITER will be equipped with networks of high performance imaging sensors able to capture visible and infrared images of the in-vessel during plasma operation. But massive data production implies to develop adapted techniques and tools for efficient imaging data processing and management. In particular, one challenge of imaging diagnostics is the automatic recognition of plasma and thermal phenomena for machine protection purposes and physics investigations using qualitative imaging techniques. To 
this end, we have pointed out the corresponding R\&D needs in both hardware and software directions. Based on report and on previous experiences, we have developed an integrated framework for phenomenological diagnostics which consists in a modular platform gathering both hardware and software components. This platform is able to perform automatic detection and recognition of plasma and thermal phenomena in real time from multi-sensor data analysis. Useful information related to detected events is automatically displayed and stored for efficient off-line data retrieval and mining. The developed software platform is daily used at Tore Supra to assist physicists and people in charge of the operation in their interpretation of the phenomenological activity. On-going work aims at integrating this platform into the plasma control system of Tore Supra to improve the PFC monitoring during operation.

\section{ACKNOWLEDGMENT}

This work was supported in part by the European Communities under the contract of Association between EURATOM, CEA and the French Research Federation for fusion studies. The views and opinions expressed herein do not necessarily reflect those of the European Commission. This work was also supported by the French Stimulus Plan 2009-2010.

\section{REFERENCES}

[1] R. Reichle, P. Andrew, G. Counsell, J.-M. Drevon, A. Encheva, G. Janeschitz, D. Johnson, Y. Kusama, B. Levesy, A. Martin, C. Pitcher, R. Pitts, D. Thomas, G. Vayakis, and M. Walsh, "Defining the infrared systems for ITER," Review of Scientific Instruments, vol. 81, no. 10, p. 10E135, 2010.

[2] R. Reichle, P. Andrew, G. Counsell, J.-M. Drevon, A. Encheva, G. Janeschitz, D. Johnson, Y. Kusama, B. Levesy, A. Martin, C. S. Pitcher, R. Pitts, D. Thomas, G. Vayakis, and M. Walsh, "Defining the infrared systems for iter," Review of Scientific Instruments, vol. 81, no. 10, pp. 10E135-10E135-5, oct 2010.

[3] P. Arena, A. Basile, R. De Angelis, L. Fortuna, G. Mazzitelli, S. Migliori, G. Vagliasindi, and M. Zammataro, "Real time monitoring of radiation instabilities in tokamak machines via CNNs," Plasma Science, IEEE Transactions on, vol. 33, no. 3, pp. 1106-1114, june 2005.

[4] P. Ghendrih, Y. Sarazin, M. Bécoulet, G. Huysmans, S. Benkadda, P. Beyer, C. Figarella, X. Garbet, and P. Monier-Garbet, "Patterns of ELM impacts on the JET wall components," Journal of Nuclear Materials, vol. 313-316, pp. 914 - 918, 2003.

[5] J. A. Alonso, P. Andrew, A. Neto, J. L. de Pablos, E. de la Cal, A. Fernandes, W. Fundamenski, C. Hidalgo, G. Kocsis, A. Murari, G. Petravich, R. A. Pitts, L. Rios, and C. Silva, "Fast visible imaging of ELM-wall interactions on JET," Journal of Nuclear Materials, vol. 390-91, pp. 797-800, 2009.

[6] A. Murari, M. Camplani, B. Cannas, D. Mazon, F. Delaunay, P. Usai, and J. Delmond, "Algorithms for the automatic identification of MARFEs and UFOs in JET database of visible camera videos," IEEE Trans. on Plasma Science, vol. 38, no. 12, pp. 3409-3418, 2010.

[7] R. Maqueda, R. Maingi, K. Tritz, K. Lee, C. Bush, E. Fredrickson, J. Menard, A. Roquemore, S. Sabbagh, and S. Zweben, "Structure of MARFEs and ELMs in NSTX," Journal of Nuclear Materials, vol. 363365, pp. 1000-1005, 2007.

[8] N. S. Love and C. Kamath, "Image analysis for the identification of coherent structures in plasma," in Proc. SPIE 6696, 66960D, 2007.

[9] U. Wenzel, K. McCormick, D. Hildebrandt, S. Klose, D. Naujoks, and H. Thomsen, "Experimental observation of MARFEs in the W7-AS stellerator," Plasma Physics and Controlled Fusion, vol. 44, no. 10, p. L57, 2002.
[10] E. Gauthier, P. Andrew, G. Arnoux, Y. Corre, and H. Roche, "Plasma wall interaction during ELMs in JET," Journal of Nuclear Materials, vol. 363-365, pp. 1026 - 1031, 2007.

[11] A. Ekedahl, J. Bucalossi, Y. Corre, E. Delchambre, G. Dunand, O. Meyer, R. Mitteau, P. Monier-Garbet, B. Pégourié, F. Rimini, F. SaintLaurent, J. Schwob, and E. Tsitrone, "Analysis of radiative disruptions in RF-heated tore supra plasmas using infrared imaging," Journal of Nuclear Materials, vol. 390-391, pp. 806 - 809, 2009.

[12] A. Roquemore, N. Nishino, C. Skinner, C. Bush, R. Kaita, R. Maqueda, W. Davis, A. Pigarov, and S. Krasheninnikov, "3D measurements of mobile dust particle trajectories in NSTX," Journal of Nuclear Materials, vol. 363-365, pp. $222-226,2007$.

[13] G. De Temmerman, J. Bacharis, M. andDowling, and S. Lisgo, "Dust creation and transport in MAST," Nuclear Fusion, vol. 50, pp. 105012 $105018(7), 2010$.

[14] S.-H. Hong, C. Grisolia, V. Rohde, P. Monier-Garbet, Tore Supra Team, and ASDEX Upgrade Team, "Temporal evolution and spatial distribution of dust creation events in tore supra and in asdex upgrade studied by ccd image analysis," Nuclear Fusion, vol. 50, no. 3, p. 035002, 2010.

[15] V. Martin, V. Moncada, G. Dunand, Y. Corre, E. Delchambre, and J.-M. Travere, "Integrated software for imaging data analysis applied to edge plasma physic and operational safety," Fusion Engineering and Design, vol. 86, no. 4-5, pp. 270-278, 2011.

[16] P. Moreau, O. Barana, S. Brémond, L. Colas, A. Ekedahl, F. SaintLaurent, C. Balorin, G. Caulier, C. Desgranges, D. Guilhem, M. Jouve, F. Kazarian, G. Lombard, L. Millon, R. Mitteau, P. Mollard, H. Roche, and J. Travere, "RF heating optimization on Tore Supra using feedback control of infrared measurements," Fusion Engineering and Design, vol. 82, no. 5-14, pp. 1030-1035, 2007.

[17] A. Herrmann, R. Drube, T. Lunt, and P. de Marne, "Real-time protection of in-vessel components in ASDEX upgrade," Fusion Engineering and Design, 2011, DOI: 10.1016/j.fusengdes.2011.02.037.

[18] Y. Corre, M. Lipa, G. Agarici, V. Basiuk, L. Colas, X. Courtois, G. Dunand, R. Dumont, A. Ekedahl, J.-L. Gardarein, C. Klepper, V. Martin, C. Moncada, V. Portafaix, F. Rigollet, R. Tawizgant, J.-M. Travère, and K. Vulliez, "Heat flux calculation and problem of flaking of boron carbide coatings on the faraday screen of the ICRH antennas during tore supra high power, long pulse operation," Fusion Engineering and Design, vol. 86, no. 4-5, pp. 429-441, 2011.

[19] R. Mitteau, J. Spruytte, S. Vallet, J. Travere, D. Guilhem, and C. Brosset, "A possible method of carbon deposit mapping on plasma facing components using infrared thermography," Journal of Nuclear Materials, vol. 363-365, pp. 206-210, 2007.

[20] V. Martin, J.-M. Travere, V. Moncada, and F. Brmond, "Towards intelligent video understanding applied to plasma facing component monitoring," Contributions to Plasma Physics, vol. 51, no. 2-3, pp. 252255, 2011.

[21] V. Martin, G. Dunand, V. Moncada, M. Jouve, and J. Travere, "New FPGA-based image-oriented acquisition and real-time processing applied to plasma facing component thermal monitoring," Review of Scientific Instruments, vol. 81, no. 10, pp. 10E113-10E113-4, oct 2010.

[22] S. Palazzo, A. Murari, G. Vagliasindi, P. Arena, D. Mazon, and A. De Maack, "Image processing with cellular nonlinear networks implemented on field-programmable gate arrays for real-time applications in nuclear fusion." Rev Sci Instrum, vol. 81, no. 8, p. 083505, 2010.

[23] J. Vega, A. Murari, A. Pereira, A. Portas, G. Ratt/'a, and R. Castro, "Overview of intelligent data retrieval methods for waveforms and images in massive fusion databases," Fusion Engineering and Design, vol. 84, no. 7-11, pp. 1916 - 1919, 2009.

[24] V. Martin, J. Travere, F. Brémond, V. Moncada, and G. Dunand, “Thermal event recognition applied to protection of tokamak plasma-facing components," IEEE Transactions on Instrumentation and Measurements, vol. 59, no. 5, pp. 1182-1191, 2010.

[25] J. L. Terry, B. LaBombard, D. Brunner, J. Payne, and G. A. Wurden, "Divertor ir thermography on ALCATOR C-Mod," Review of Scientific Instruments, vol. 81, no. 10, pp. 10E513-10E513-4, oct 2010.

[26] G. Hommen, M. De Baar, P. Nuij, G. McArdle, R. Akers, and M. Steinbuch, "Optical boundary reconstruction of tokamak plasmas for feedback control of plasma position and shape." Review of Scientific Instruments, vol. 81 , no. 11 , p. $113504,2010$. 